\title{
Acute myeloid leukemia harboring $t(8 ; 21)(q 22 ; q 22)$ : a heterogeneous disease with poor outcome in a subset of patients unrelated to secondary cytogenetic aberrations
}

\author{
Pei Lin ${ }^{1}$, Lei Chen ${ }^{1}$, Rajyalakshmi Luthra ${ }^{1}$, Sergej N Konoplev ${ }^{1}$, Xuemei Wang ${ }^{2}$ \\ and L Jeffrey Medeiros ${ }^{1}$ \\ ${ }^{1}$ Department of Hematopathology, The University of Texas, MD Anderson Cancer Center, Houston, TX, USA \\ and ${ }^{2}$ Department of Biostatistics, The University of Texas, MD Anderson Cancer Center, Houston, TX, USA
}

\begin{abstract}
Acute myeloid leukemia with $\mathrm{t}(8 ; 21)(q 22 ; q 22)$ is a distinct type of leukemia considered to have a favorable prognosis. However, some patients rapidly succumb to disease despite chemotherapy. We studied 56 patients with acute myeloid leukemia associated with $t(8 ; 21)$ and correlated clinicopathologic, cytogenetic and molecular findings with outcome to identify markers of prognosis. In a subset of patients, we also assessed the status of the $c-K I T, F L T 3$ and RAS genes. There were 31 men and 25 women, with a median age of 38 years (range 4-76). The follow-up period ranged from 17 to 104 months (median 52). At the last follow-up, 29 patients had died, 25 patients were in complete remission and two patients were alive with disease. The median survial was 38 months. The 5-year overall survival rate of newly diagnosed patients was $56 \%$. Most patients (39/56, 70\%) had chromosomal aberrations in addition to $t(8 ; 21)$, with loss of a sex chromosome (39\%) being most common followed by del(9q)(q21-22) (11\%) and trisomy 8 (7\%). These aberrations, however, did not predict survival. C-KIT (D816V or D816Y), FLT3 (ITD or D835) and RAS mutations were detected in 26, 10 and $7 \%$, respectively, of cases assessed. The 5-year overall survival rate of patients with mutated leukemia was $20 \%$. No mutations were observed in three patients who died within 7 months of diagnosis. Leukocytosis or CD56 expression did not correlate with a poor survival nor did the levels of CD19 expression predict $c-K I T$ mutation status. We conclude that acute myeloid leukemia associated with $t(8 ; 21)$ is a heterogeneous disease with poor survival in a subset of patients unrelated to common secondary cytogenetic aberrations.
\end{abstract}

Modern Pathology (2008) 21, 1029-1036; doi:10.1038/modpathol.2008.92; published online 6 June 2008

Keywords: t(8;21); cytogenetics; $c-K I T$ mutation

The $\mathrm{t}(8 ; 21)(\mathrm{q} 22 ; \mathrm{q} 22)$ is the most common cytogenetic abnormality in acute myeloid leukemia (AML), occurring in up to $12 \%$ of patients. ${ }^{1}$ As a result of $\mathrm{t}(8 ; 21)$, the ETO (MTG8) gene on chromosome 8 is fused to the AML1 (RUNX1) gene on chromosome 21, producing a novel chimeric gene, AML1-ETO. The AML1 gene encodes the alpha subunit of core-binding factor (CBF) that is essential for normal hematopoiesis. AML1-ETO fusion gene disrupts the $\mathrm{CBF}$ transcription complex, thus initiating the first step of leukemogenesis. ${ }^{2}$ The AML1-ETO fusion protein is a multifunctional cellular protein that affects cell differentiation, proliferation, apoptosis and self-renewal. ${ }^{3}$ Evidence suggests that additional

Correspondence: Dr P Lin, MD, Department of Hematopathology, the University of Texas MD Anderson Cancer Center, 1515 Holcombe Blvd, Houston, TX 77003, USA.

E-mail: peilin@mdanderson.org

Received 09 November 2007; revised 03 April 2008; accepted 05 April 2008; published online 6 June 2008 cytogenetic aberrations may act synergistically with AML-ETO in leukemogenesis. ${ }^{3}$

AML with $\mathrm{t}(8 ; 21)$, similar to AML with inv(16) or $\mathrm{t}(15 ; 17)$, is generally considered a disease with an overall favorable prognosis characterized by a higher response rate and longer median survival compared with other types of AML. This concept is adopted in the current World Health Organization Classification. ${ }^{4}$ Of the two references cited by the WHO classification, both published in $1998,{ }^{5,6}$ one was a British study of 1612 AML patients in which 122 patients had AML with t(8;21). The 5-year overall survival (OS) rate of patients with $\mathrm{t}(8 ; 21)$ was $69 \%$ compared to $42 \%$ in AML patients with a normal karyotype. $^{5}$ In another study, ${ }^{6}$ the survival of patients with $t(8 ; 21)$, inv(16) and $t(15 ; 17)$ were analyzed as a group of 57 cases from a cohort of 285 AML patients. The estimated 5-year survival rate of this group was $50 \%$ compared to $32 \%$ for AML with a normal karyotype. 
We have observed variable clinical outcomes in AML associated with $\mathrm{t}(8 ; 21)$, with some patients rapidly succumbing to the disease within a few months of diagnosis despite chemotherapy. An older study of 41 patients with $t(8 ; 21)$ treated in our hospital during the period of 1975-1987 found that the median survival was 17 months (range: $0.5-$ 138 ) with a 3 -year survival rate of $31 \% .^{7}$ A more recent study of 31 patients published by a different group made a similar observation with a median OS of 12.5 months. $^{8}$

In patients with poor outcome, several adverse prognostic indicators have been suggested as possible explanations. Among these are certain secondary cytogenetic aberrations, leukocytosis, CD56 expression and extramedullary manifestations. Review of the literature found that some suggested indicators, most notably $\mathrm{CD}^{2} 6^{9}$ and extramedullary involvement,$^{10}$ were identified in a single or relatively small studies; others were based on the patients treated with different protocols ${ }^{11-14}$ or included secondary leukemia, ${ }^{15}$ rendering it difficult to compare and draw consistent conclusions. This is particularly true regarding implications of additional cytogenetic aberrations. Loss of a sex chromosome and $\operatorname{del}(9 q)$ have been reported to adversely, ${ }^{16}$ favorably $^{1,17,18}$ or marginally affect the survival of patients with $\mathrm{t}(8 ; 21) .{ }^{5,15,19,20}$

Earlier studies were largely based on the observation of patients treated with conventional chemotherapy regimens with idarubicin and cytarabine (ara-C). A new therapeutic regimen based on the combination of fludarabine and ara-C has been introduced in the past decade. Bone marrow transplantation has increasingly become a part of the therapeutic approach. Molecular genetic investigation also revealed that receptor tyrosine kinase (RTK) gene mutations may impact outcome in patients with AML with $t(8 ; 21) .{ }^{21-24}$

To further investigate the role of previously identified prognostic indicators in AML with $t(8 ; 21)$, particularly cytogenetic findings, in light of the advances in the past decade, we examined 56 cases of AML with $t(8 ; 21)$ diagnosed and treated in our hospital from May 1995 to July 2005. We reviewed the cytogenetic data of each case in the context of the overall clinicopathologic presentation and correlated the results with overall survival. We also selectively analyzed for $c$-KIT gene mutations in a subset of cases and reviewed FLT3 and RAS gene status in those cases that were analyzed routinely at the time of initial evaluation at our hospital.

\section{Materials and methods}

\section{Patients Selection}

We searched the files of our hospital for patients with AML carrying t(8;21)(q22;q22) from 1995 to 2005. Diagnosis and classification were based on WHO classification criteria. ${ }^{4}$ Peripheral blood, bone marrow aspirate smears and biopsy specimens were reviewed. Clinical information was obtained from the review of medical records. All samples were obtained under an Institutional Review Board approved protocol.

The OS and impact of additional cytogenetic aberrations on survival were analyzed using the Kaplan-Meier method. Patients with three or more numerical or structural abnormalities were defined as having a complex karyotype. A multivariate Cox proportional hazard model was used to analyze the impact of age, gender, leukocyte count as well as CD19 or CD56 expression on survival. Survival curves were plotted using Statistica 6 software (StatSoft, Tulsa, OK).

\section{Conventional Cytogenetics and FISH Studies}

Metaphase cells from bone marrow aspirate samples were cultured for 24 and $48 \mathrm{~h}$ using previously described methods. ${ }^{25}$ The slides were prepared and G-banded by conventional methods. A minimum of two metaphases was required for the presence of a clonal abnormality. Fluorescence in situ hybridization (FISH) was performed on a fresh dropped slide from harvested bone marrow aspirate specimen or a G-banded slide using a commercially available probe (Vysis, Downers Grove, IL). ${ }^{25}$ A total of 200 interphases or 10 metaphases were analyzed. (Carl Zeiss, Thornwood, NY).

\section{RT-PCR}

Following reverse transcription, AML1-ETO transcripts were analyzed by qualitative or quantitative TaqMan RT-PCR (Ipsogen, Marseille, France). The Kasumi-1 and HL-60 cell lines were used as positive and negative controls, respectively. Sequencing analysis of exons 8 and 17 of the $C$-KIT gene was performed using genomic DNA extracted from bone marrow aspirate samples and methods described previously. ${ }^{23}$ Methods for detecting FLT3 internal tandem repeat (ITD) or D835 point mutation and an assay to detect RAS mutations were described previously. ${ }^{26,27}$

\section{Flow Cytometry Immunophenotyping}

Peripheral blood or bone marrow aspirate specimens were collected in EDTA tubes. Three- or four-color flow cytometric analysis was performed using FACScan or FACSCaliber cytometer (BD Biosciences, San Jose, CA) as described previously. ${ }^{25}$ A panel of monoclonal antibodies was performed, including CD3, CD7, CD10, CD13, CD19, CD20, CD34, CD64 and CD117, as well as HLA-DR and TdT (terminal deoxynucleotidyl tansferase). CD56 was also performed in a subset of cases. The percentage of blasts expressing 
CD19 or CD56 was recorded and used for statistical analysis.

\section{Results}

We initially identified 66 patients diagnosed with AML associated with $\mathrm{t}(8 ; 21) ; 11$ patients were excluded from the study, including seven who had therapy-related AML, two who had blast crisis of chronic myelogenous leukemia, and one who declined treatment. The study group included 56 patients with leukemia-38 newly diagnosed, 17 relapsed and one primary refractory. The clinicopathologic features of the 56 patients are summarized in Table 1.

Most patients $(n=39,70 \%)$ had chromosomal aberrations in addition to $t(8 ; 21)$. The frequency of additional aberrations did not differ significantly between newly diagnosed cases vs relapsed/ refractory cases: 25/38 (66\%) vs 14/18 (78\%); $P=1.00$ (Fisher's exact test). Loss of a sex chromosome $(n=22,39 \%)$ was most common followed by del(9q)(q21-22) $(n=6,11 \%)$ and trisomy $8(n=4,7 \%)$. Seven patients had a complex karyotype. No individual secondary chromosomal amoralities were more common in either newly diagnosed or relapsed/refractory cases (Fisher's exact test).

For the newly diagnosed patients, most (68\%) were treated with a regimen consisting of a combination of fludarabine and ara-C and the complete remission rate was $95 \%$. Fourteen (37\%) patients subsequently relapsed and were reinduced with various salvage protocols including bone marrow transplantation in nine patients. No patient received bone marrow transplantation during the first remission. All relapsed/refractory patients referred to our hospital were initially treated with conventional chemotherapy regimens with idarubicin or daunorubicin with ara-C at the outside hospitals.

The follow-up period ranged from 17 to 104 months, with a median of 52 months. At time of last follow-up, 29 patients died, 25 patients were in complete remission and two patients were alive with disease. The median survival was 38 months. The 5-year OS rate was $47 \%$, with $95 \%$ confidence interval (CI) of $35-62 \%$ (Figure 1). The 3-year and 5 -year survival rate of 38 newly diagnosed patients was $63 \%$ (95\% CI: $54-70 \%)$ and $56 \%$ (95 CI: 48-64\%), respectively. Age and gender did not predict OS, nor did total leukocyte count using a cutoff of either 20 or $30 \mathrm{k} / \mu \mathrm{l}(P=0.5)$.

\section{Impact of Additional Cytogenetic Aberrations on OS}

First, all patients were divided into two subgroups for the analysis of prognosis: group 1: isolated $\mathrm{t}(8 ; 21)$ and group 2 : $\mathrm{t}(8 ; 21)$ plus other cytogenetic aberrations. The 5-year OS rates for groups
Table 1 Clinicopathological features of 56 patients with acute myeloid leukemia harboring $\mathrm{t}(8 ; 21)$

\begin{tabular}{|c|c|}
\hline Total number of cases & 56 \\
\hline De novo & 38 \\
\hline Relapsed/refractory & 18 \\
\hline \multicolumn{2}{|l|}{ Gender } \\
\hline Male/female & $31(20) / 25(18)^{\mathrm{a}}$ \\
\hline \multicolumn{2}{|l|}{ Age (years) } \\
\hline$<15$ & $(2)^{\mathrm{a}}$ \\
\hline $16-60$ & $50(32)^{\mathrm{a}}$ \\
\hline$>60$ & $(4)^{\mathrm{a}}$ \\
\hline \multicolumn{2}{|l|}{$W B C(k / \mu L)$} \\
\hline Median & 11.7 \\
\hline Range & $4.7-92$ \\
\hline \multicolumn{2}{|l|}{ CD56 } \\
\hline$>30 \%$ & 16 \\
\hline $15-20 \%$ & 4 \\
\hline $0-15 \%$ & 9 \\
\hline \multicolumn{2}{|l|}{ Extramedullary myeloid tumor } \\
\hline Total & 9 \\
\hline At presentation & 1 \\
\hline At Relapse & $8^{\mathrm{b}}$ \\
\hline \multicolumn{2}{|l|}{ Secondary cytogenetic aberrations } \\
\hline Total & $39 / 56$ \\
\hline De novo & $25 / 38$ \\
\hline Relapsed/refractory & $14 / 18$ \\
\hline$-\mathrm{X}$ & $7(6)^{\mathrm{a}}$ \\
\hline$-\mathrm{Y}$ & $15(10)^{\mathrm{a}}$ \\
\hline $\operatorname{Del}(9 q)$ & $6(5)^{\mathrm{a}}$ \\
\hline Complex & $7(3)^{a}$ \\
\hline \multicolumn{2}{|l|}{ Therapy } \\
\hline \multicolumn{2}{|l|}{ De novo } \\
\hline FLAG & 26 \\
\hline CATG & 8 \\
\hline Idarubicin or daunorubicin+ara-C & 2 \\
\hline Daunorubicin+ara-C+etopside & $2^{\mathrm{c}}$ \\
\hline Allo-BMT & $9^{d}$ \\
\hline \multicolumn{2}{|l|}{ Relapsed/refractory } \\
\hline Idarubicin+ara-C & 17 \\
\hline Daunorubicin+ara-C & 1 \\
\hline Allo-BMT & 6 \\
\hline
\end{tabular}

Allo-BMT, allogeneic bone marrow transplantation; CATG, cyclophosphamide, ara-C, topotecan, G-CSF; FLAG, fludarabine, ara-C and G-CSF.

${ }^{\mathrm{a}}$ Cases in parenthesis are de novo cases.

${ }^{\mathrm{b}}$ Three of the 8 cases were originally diagnosed at our hospital.

${ }^{\mathrm{C}}$ Protocol for pediatric patients.

${ }^{\mathrm{d}}$ BMT employed after relapse.

1 and 2 were $47 \%$ (CI: $30-76 \%$ ) and $46 \%$ (CI: $32-66 \%)$, respectively $(P=0.81)$ (Figure 2). Exclusion of refractory and relapsed patients from the analysis yielded a similar result $(P=0.19)$ Comparison between patients with a complex karyotype and those with isolated $t(8 ; 21)$ also found no significant difference in survival for the entire cohort $(P=0.91)$ (Figure 3) or newly diagnosed patients $(P=0.25)$. 


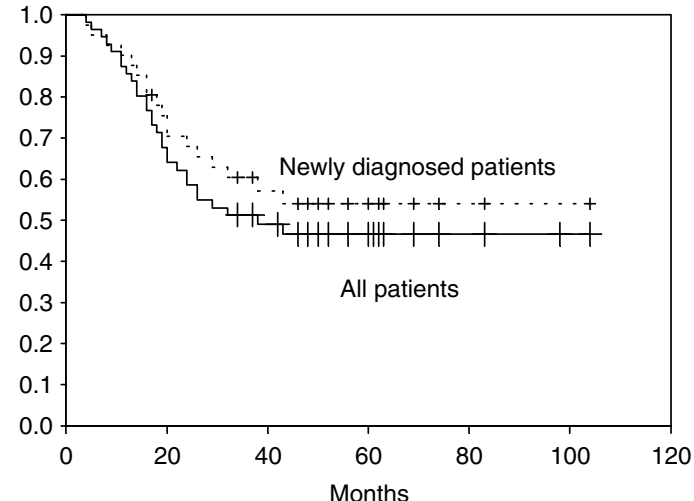

Figure 1 Overall survival of all 56 patients and 38 newly diagnosed patients with acute myeloid leukemia harboring $\mathrm{t}(8 ; 21)(\mathrm{q} 22 ; \mathrm{q} 22)$.

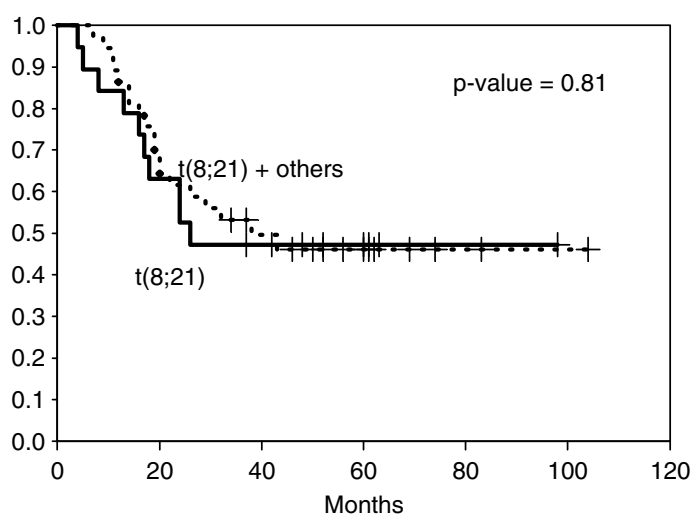

Figure 2 Overall survival of patients with isolated $t(8,21)(n=17)$ vs patients with $\mathrm{t}(8 ; 21)$ and additional aberrations $(n=39)$.

\section{Impact of Loss of a Sex Chromosome or del(9q) on OS}

Loss of a sex chromosome was found in 22 (39\%), chromosome $\mathrm{Y}$ in $15(27 \%)$ and chromosome $\mathrm{X}$ in seven $(13 \%)$ patients. The age of the affected patients ranged from 16 to 69 (median 53 years). The OS was not significantly different between patients with loss of Y chromosome and other male patients $(P=0.14)$ (Figure 4). Similarly, OS was not significantly different between patients with loss of an X chromosome and other female patients $(P=0.18)$ (Figure 5). A similar finding was observed when six patients with $\operatorname{del}(9)$ were compared with patients without $\operatorname{del}(9)(P=0.82)$ (Figure 6).

\section{Impact of CD19 Or CD56 Expression on OS}

The percentages of CD19-positive blasts ranged from 0 to $94 \%$ with a median of $58 \%$. CD56 was analyzed only in 29 (18 newly diagnosed) patient samples and the percentage of blasts positive for the marker ranged from 0 to $87 \%$. Seven $(39 \%)$ patient had CD56 expression in $30 \%$ of leukemic cells. Expression levels of CD56 or CD19 did not predict OS based on the fitted univariate Cox proportional hazards model ( $P=1.0$ and $P=0.937$, respectively).

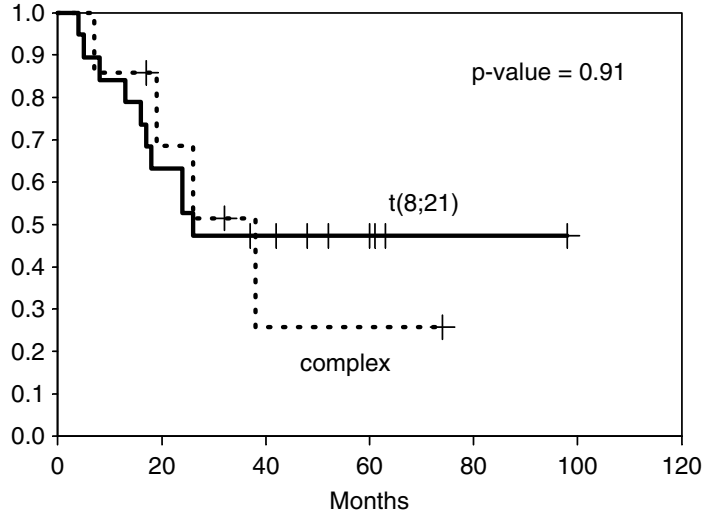

Figure 3 Overall survival of patients with isolated $\mathrm{t}(8 ; 21 ; n=17)$ vs patients with a complex karyotype $(n=7)$.

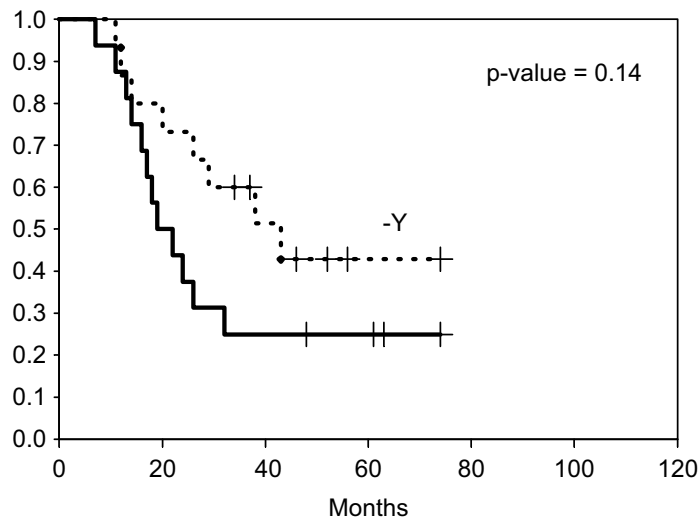

Figure 4 Overall survival of patients with loss of $Y$ chromosome $(n=15)$ vs other male patients $(n=16)$.

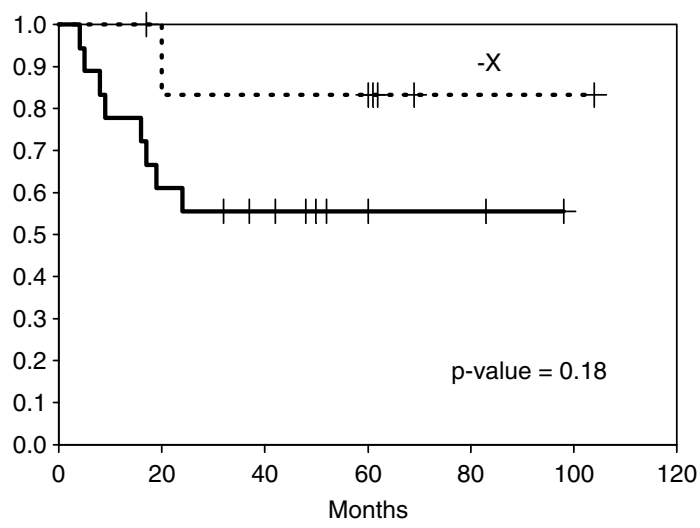

Figure 5 Overall survival of patients with loss of an $\mathrm{X}$ chromosome $(n=7)$ vs other female patients $(n=17)$.

\section{c-KIT, FLT and RAS Gene Mutations Compared with OS}

C-KIT status was analyzed in 19 cases, 10 from patients who survived $>41$ months and nine from those who survived $<14$ months. Five cases were positive, with only one patient in the prolonged survival group; one case carried an isolated $t(8 ; 21)$ 


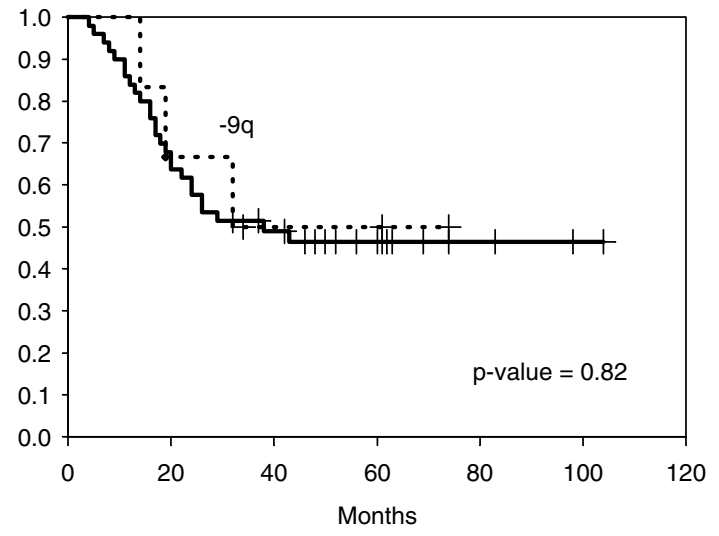

Figure 6 Overall survival of patients with deletion of chromosome 9q $(n=6)$ vs those without the deletion $(n=50)$.

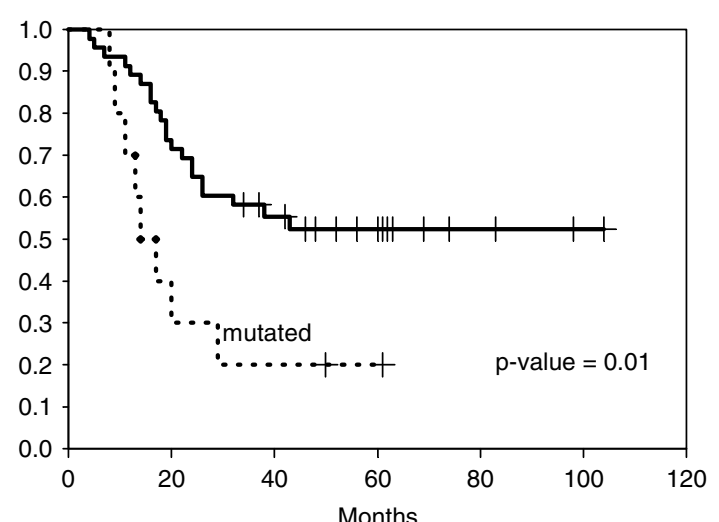

Figure 7 Overall survival of patients with mutated $c$-KIT, FLT3 or $R A S$ genes vs remainder of study patients.

and four others had additional aberrations. CD19 expression varied from absent $(<1 \%)$ in one case to partial $(28-43 \%)$ in three cases and most cells in one case $(79 \%)$.

FLT3 and RAS mutations were identified in 3/29 $(10 \%)$ and $2 / 27(7 \%)$ cases assessed. No case had both FLT3 and RAS mutations. FLT3 mutations were of internal tandem repeat type in two cases and D835 point mutation type in one case. Four of these five patients died within 25 months of diagnosis.

When cases with any mutation were grouped and compared with the remainder of patients in the study group, the 5-year OS rate of patients with mutated AML was $20 \%$ (CI: 6-69\%) compared to $52 \%$ (CI: $39-70 \%)(P=0.01)$ for the remainder of patients (Figure 7). A similar analysis performed for the newly diagnosed cases yielded a similar result $(P=0.007)$.

\section{Discussion}

As it was first described by Rowley ${ }^{28}$ in 1973, AML with $\mathrm{t}(8 ; 21)$ has been recognized as a distinct subtype of AML with a more favorable prognosis. Despite the high complete remission rate and OS, the clinical outcome of these patients is heterogeneous. In this study, eight $(14 \%)$ patients died within 1 year and $24(43 \%)$ patients died within 2 years of diagnosis. In sum, $93 \%$ of patients who eventually succumbed to disease died within 3 years of diagnosis and the 5-year OS of newly diagnosed patients was $56 \%$.

Adverse prognostic factors reported in AML with $t(8 ; 21)$ included leukocytosis, certain secondary cytogenetic aberrations, extramedullary manifestations, CD56 expression and, more recently, c-KIT mutation. Many earlier studies were limited by small sample size or heterogeneous patient composition resulting in conflicting data, especially regarding the role of secondary cytogenetic aberrations. Table 2 summarizes the published data on the subject of additional cytogenetic aberrations in AML with $t(8 ; 21)$. Notably, the loss of a sex chromosome and deletion of chromosome $9 \mathrm{q}$ are most common and their impact on survival is controversial. ${ }^{1,5,15,17-20}$

In this study, we confirmed that the loss of a sex chromosome and del(9q) were common, in 22 (39\%) and six (11\%) cases, respectively, and these aberrations did not correlate with poorer clinical outcome. These results are in keeping with the observations by other investigators who had $>100$ cases in their respective study series. ${ }^{5,12,13,15}$ These series demonstrated no deleterious effect of loss of a sex chromosome or $\operatorname{del}(9 \mathrm{q})$ on the outcome of patients with $t(8 ; 21)$. The strength of our series is that the large majority of newly diagnosed patients were treated similarly in a single institution instead of multiple different protocols as in earlier large series, allowing us to assess the prognostic factors across different subgroups despite the relatively small cohort size. The del(9q) was identified as a negative $^{16}$ or positive ${ }^{18}$ prognostic factor in one study each. In the former study, 7 of 50 patients with $\operatorname{del}(9 q)$ had a median OS of 12.5 months. ${ }^{16}$ In the latter, 8 of 51 patients with an abnormal long arm of chromosome 9 (two with translocations and six with partial deletions) had a longer event-free survival of unspecified time. ${ }^{18}$ These two studies highlight the limitation of small study series and difficulty comparing data from various study series.

In an earlier study from our institution published in $1998,{ }^{7}$ marked leukocytosis, defined as a white cell count of $>20 \mathrm{k} / \mu \mathrm{l}$, negatively impacted patient survival. This finding was not confirmed in this study. It is possible that this may be explained by changes in the treatment regimen. Most patients in this study were treated with fludarabine and ara-C. Fludarabine is a purine analog that inhibits DNA and enhances intracellular concentration of the active metabolite of ara-C. ${ }^{29}$ Our study is the only one that focused on patients with AML associated with $t(8 ; 21)$ treated primarily with fludarabine and ara- $C$ rather than the conventional regimens. The study by Appelbaum et al, ${ }^{15}$ though also comparing a regimen based on fludarabine and ara-C with other 
Table 2 Summary of the common secondary cytogenetic aberrations in acute myeloid leukemia with $\mathrm{t}(8 ; 21)$ and their effects on survival reported in the literature

\begin{tabular}{|c|c|c|c|c|}
\hline Authors & Year published & $\begin{array}{l}\text { Total number } \\
\text { of cases }\end{array}$ & $\operatorname{Del}(9 q)$ & Loss of $X$ or $Y$ \\
\hline Appelbaum et $a l^{15 \text { a }}$ & 2006 & 174 & 25 , no effect & -Y: 53; -X: 28, no effect \\
\hline Marcucci et al ${ }^{12}$ & 2005 & 144 & 25 , no effect & $\begin{array}{l}-\mathrm{Y}: 46 ;-\mathrm{X}: 22 \\
\text { no effect }\end{array}$ \\
\hline Schlenk et al ${ }^{14}$ & 2004 & 185 & 31 , no effect & $-Y: 58$; worse, $-X: 31$, no effect \\
\hline Nishii et $a 1^{19}$ & 2003 & 94 & 10 , no effect & 27 , no effect \\
\hline Byrd et al ${ }^{11}$ & 2002 & 81 & 18 , no effect & 38 , no effect \\
\hline Nguyen et $a l^{13}$ & 2002 & 154 & 13 , no effect & 67 , no effect \\
\hline Byrd et al ${ }^{11}$ & 2002 & 81 & 18, no effect & 38 , no effect \\
\hline Rege et $a l^{18}$ & 2000 & 50 & $8^{\mathrm{b}}$, favorable & 17 , worse \\
\hline Grimwade et $a l^{5 \mathrm{c}}$ & 1998 & 122 & 9 , no effect & 57 , no effect \\
\hline Schoch et $a l^{16}$ & 1996 & 51 & 7 , worse & 31 , no effect \\
\hline Haferlach et $a l^{1}$ & 1996 & 41 & - & $\begin{array}{l}-\mathrm{Y}: 12,-\mathrm{X}: 4, \\
\text { favorable }\end{array}$ \\
\hline
\end{tabular}

Only studies with $>40$ cases are included in the table.

${ }^{\mathrm{a}}$ Of the 174 patients, 79 were newly diagnosed, 28 were secondary and 67 were unknown.

${ }^{\mathrm{b}}$ Included two cases with translocations and six cases with partial deletions of the long arm of a chromosome 9.

${ }^{\mathrm{c}} \mathrm{A}$ total of 118 cases were newly diagnosed and four were secondary cases.

Bold text indicates prognostic significance.

therapeutic regimens, included a large number of secondary leukemias; and analyzed 53 patients with $\mathrm{t}(8 ; 21)$ or inv(16) as a single category of core-binding leukemia. The number of cases with $t(8 ; 21)$ and treated with fludarabine was not specified. Given that the OS of patients with $t(8 ; 21)$ is inferior to that of inv(16),,$^{15}$ this approach clearly has limitations.

The survival rate of our patients was comparable to most other reports, ${ }^{12,14,15}$ but was lower than a British study-56 vs $69 \% .^{5}$ This is likely due to less strict criteria for complete remission, as acknowledged by the authors, and inclusion of patients treated with bone marrow transplantation at the first remission in the British study. Further analysis could not confirm that CD56 expression was associated with poor survival in our group though only 29 patients were analyzed for this marker. As only one study patient presented with an extramedullary myeloid tumor, we were unable to determine its impact on patient outcome. However, we did observe that this phenomenon was more common in patients with relapsed disease (16\%).

Rare studies in the past have disputed the concept that AML with $t(8 ; 21)$ was a favorable group compared with other AML with maturation. ${ }^{9,30,31}$ The dichotomy is evident in a recent Korean study, which demonstrated no survival difference between AML with $\mathrm{t}(8 ; 21)$ and without $\mathrm{t}(8 ; 21){ }^{8}$ It is possible that the discordance may be due to the difference in the genetic makeup of the study patients or regimens employed. Only one study published so far revealed a shorter survival of nonwhite patients than white patients in AML associated with $t(8 ; 21)$ when certain secondary cytogenetic aberrations are present. ${ }^{12}$ More studies are needed to investigate whether survival differences in different ethnic groups are related to biological differences or chemosensitivity.

Recent studies have emphasized the role of RTK gene mutations, such as FLT3/ITD and $c-K I T / D 816 \mathrm{~V}$, in leukemogenesis. The frequency of $c-K I T$ mutations ranges from 24 to $39 \%$ in AML with $t(8 ; 21)$ compared with less than $2 \%$ in AML cases overall, and is reported to negatively impact OS and eventfree survival. ${ }^{24}$ Similar results were reported in pediatric patients with t(8;21)-AML. ${ }^{32}$ FLT3 mutations have been detected in up to $30 \%$ of AML cases and correlate with a poor prognosis. ${ }^{24,33,34}$ In our experience, FLT3 and RAS mutations are uncommon in AML with $\mathrm{t}(8 ; 21)-10 \%$ or less of cases assessed were positive for FLT3 or RAS mutations. By contrast, $C$-KIT mutation was identified in a quarter of patients, more often in the group with short survival. In this study, we were unable to show that c-KIT mutation impacts survival. However, this may be attributable to sample size. We did show that patients with mutated AML (c-KIT, FLT3 or RAS) had a significantly worse prognosis than the rest in the study. The single patient who was still alive and free of disease 61 months after diagnosis, despite having $c-K I T$ mutation, had multiple relapses requiring allogeneic bone marrow transplantation. On the other hand, three patients with extremely short survival $(<7$ months) did not carry $C-K I T$ (D816V/D816Y), FLT3 (ITD or D835) or RAS mutations and two of these cases had isolated $t(8 ; 21)$. Dicker et $a l^{35}$ found trisomy 13 as a mechanism of FLT3 overexpression in AML as FLT3 is located on chromosome 13 . We did not find any case with trisomy 13 in our study. These findings suggest that 
other molecular genetic factors, such as those affecting chemosensitivity, most likely impact patient survival.

De et $a l^{36}$ reported that the absence of CD19 expression correlates with $c$-KIT mutation. However, we did not confirm this finding in our study. Of the five patients with $C$-KIT mutation, the expression levels of CD19 in the leukemic blasts varied from absent to high suggesting that CD19 is not reliable as a surrogate marker for $c$-KIT mutation.

In summary, the outcome of patients with AML associated with $\mathrm{t}(8 ; 21)$ and treated with fludarabinebased regimens is variable and not predictable by conventional cytogenetic data. While cases with mutated $C-K I T D 816 V / D 816 Y, F L T 3$ or $R A S$ as a group had a worse survival, several patients with exceptionally short survival did not carry these mutations, suggesting that other molecular factors such chemosensitivity are likely implicated and need to be further explored. Cases with or without isolated $\mathrm{t}(8 ; 21)$ are both susceptible to $C$-KIT mutation and CD19 expression level does not reliably predict $C$-KIT status.

\section{References}

1 Haferlach T, Bennett JM, Loffler $\mathrm{H}$, et al. Acute myeloid leukemia with translocation $(8 ; 21)$. Cytomorphology, dysplasia and prognostic factors in 41 cases. AML Cooperative Group and ECOG. Leuk Lymphoma 1996;23:227-234.

2 Peterson LF, Zhang DE. The 8;21 translocation in leukemogenesis. Oncogene 2004;23:4255-4262.

3 Peterson LF, Boyapati A, Ahn EY, et al. Acute myeloid leukemia with the 8q22;21q22 translocation: secondary mutational events and alternative $\mathrm{t}(8 ; 21)$ transcripts. Blood 2007;110:799-805.

4 Brunning RD, Matutes E, Flandrin G, et al. Acute myeloid leukaemia with recurrent genetic abnormalities. In: Jaffe ES, Harris NL, Stein H, Vardiman JW (eds). World Health Organization Classification of Tumours. Pathology and Genetics. Tumours of Haematopoietic and Lymphoid Tissues. IARC Press: Lyon, France, 2001, pp 81-82.

5 Grimwade D, Walker H, Oliver F, et al. The importance of diagnostic cytogenetics on outcome in AML: analysis of 1,612 patients entered into the MRC AML 10 trial. The Medical Research Council Adult and Children's Leukaemia Working Parties. Blood 1998;92:2322-2333.

6 Bloomfield CD, Lawrence D, Byrd JC, et al. Frequency of prolonged remission duration after high-dose cytarabine intensification in acute myeloid leukemia varies by cytogenetic subtype. Cancer Res 1998;58: 4173-4179.

7 O’Brien S, Kantarjian HM, Keating M, et al. Association of granulocytosis with poor prognosis in patients with acute myelogenous leukemia and translocation of chromosomes 8 and 21. J Clin Oncol 1989;7: 1081-1086.

8 Lee KW, Choi IS, Roh EY, et al. Adult patients with $t(8 ; 21)$ acute myeloid leukemia had no superior treatment outcome to those without $t(8 ; 21)$ : a single institution's experience. Ann Hematol 2004;83: 218-224.

9 Baer MR, Stewart CC, Lawrence D, et al. Expression of the neural cell adhesion molecule CD56 is associated with short remission duration and survival in acute myeloid leukemia with $t(8 ; 21)(q 22 ; q 22)$. Blood 1997;90:1643-1648.

10 Byrd JC, Weiss RB, Arthur DC, et al. Extramedullary leukemia adversely affects hematologic complete remission rate and overall survival in patients with $\mathrm{t}(8 ; 21)(\mathrm{q} 22 ; \mathrm{q} 22)$ : results from Cancer and Leukemia Group B 8461. J Clin Oncol 1997;15:466-475.

11 Byrd JC, Mrozek K, Dodge RK, et al. Pretreatment cytogenetic abnormalities are predictive of induction success, cumulative incidence of relapse, and overall survival in adult patients with de novo acute myeloid leukemia: results from Cancer and Leukemia Group B (CALGB 8461). Blood 2002;100:4325-4336.

12 Marcucci G, Mrozek K, Ruppert AS, et al. Prognostic factors and outcome of core binding factor acute myeloid leukemia patients with $\mathrm{t}(8 ; 21)$ differ from those of patients with inv(16): a Cancer and Leukemia Group B study. J Clin Oncol 2005;23:5705-5717.

13 Nguyen S, Leblanc T, Fenaux P, et al. A white blood cell index as the main prognostic factor in $\mathrm{t}(8 ; 21)$ acute myeloid leukemia (AML): a survey of 161 cases from the French AML Intergroup. Blood 2002;99: 3517-3523.

14 Schlenk RF, Benner A, Krauter J, et al. Individual patient data-based meta-analysis of patients aged 16 to 60 years with core binding factor acute myeloid leukemia: a survey of the German Acute Myeloid Leukemia Intergroup. J Clin Oncol 2004;22:3741-3750.

15 Appelbaum FR, Kopecky KJ, Tallman MS, et al. The clinical spectrum of adult acute myeloid leukaemia associated with core binding factor translocations. Br J Haematol 2006;135:165-173.

16 Schoch C, Haase D, Haferlach T, et al. Fifty-one patients with acute myeloid leukemia and translocation $\mathrm{t}(8 ; 21)(\mathrm{q} 22 ; \mathrm{q} 22)$ : an additional deletion in $9 \mathrm{q}$ is an adverse prognostic factor. Leukemia 1996;10: 1288-1295.

17 Hann IM, Webb DK, Gibson BE, et al. MRC trials in childhood acute myeloid leukaemia. Ann Hematol 2004;83(Suppl 1):S108-S112.

18 Rege K, Swansbury GJ, Atra AA, et al. Disease features in acute myeloid leukemia with $t(8 ; 21)(q 22 ; q 22)$. Influence of age, secondary karyotype abnormalities, CD19 status, and extramedullary leukemia on survival. Leuk Lymphoma 2000;40:67-77.

19 Nishii K, Usui E, Katayama N, et al. Characteristics of $\mathrm{t}(8 ; 21)$ acute myeloid leukemia (AML) with additional chromosomal abnormality: concomitant trisomy 4 may constitute a distinctive subtype of $t(8 ; 21)$ AML. Leukemia 2003;17:731-737.

20 Billstrom R, Johansson B, Fioretos $\mathrm{T}$, et al. Poor survival in $\mathrm{t}(8 ; 21)(\mathrm{q} 22 ; \mathrm{q} 22)$-associated acute myeloid leukaemia with leukocytosis. Eur J Haematol 1997;59:47-52.

21 Cairoli R, Beghini A, Grillo G, et al. Prognostic impact of c-KIT mutations in core binding factor leukemias: an Italian retrospective study. Blood 2006; 107:3463-3468.

22 Kuchenbauer F, Schnittger S, Look AT, et al. Identification of additional cytogenetic and molecular genetic abnormalities in acute myeloid leukaemia with $\mathrm{t}(8 ; 21) /$ AML1-ETO. Br J Haematol 2006;134:616-619. 
23 Lasa A, Carricondo MT, Carnicer MJ, et al. A new D816 c-KIT gene mutation in refractory AML1-ETO leukemia. Haematologica 2006;91:1283-1284.

24 Schnittger S, Kohl TM, Haferlach T, et al. KIT-D816 mutations in AML1-ETO-positive AML are associated with impaired event-free and overall survival. Blood 2006;107:1791-1799.

25 Yin CC, Medeiros LJ, Glassman AB, et al. $\mathrm{t}(8 ; 21)(\mathrm{q} 22 ; \mathrm{q} 22)$ in blast phase of chronic myelogenous leukemia. Am J Clin Pathol 2004;121:836-842.

26 Hirsch-Ginsberg C, LeMaistre AC, Kantarjian H, et al. RAS mutations are rare events in Philadelphia chromosome-negative/bcr gene rearrangement-negative chronic myelogenous leukemia, but are prevalent in chronic myelomonocytic leukemia. Blood 1990;76: 1214-1219.

27 Lin P, Jones D, Medeiros LJ, et al. Activating FLT3 mutations are detectable in chronic and blast phase of chronic myeloproliferative disorders other than chronic myeloid leukemia. Am J Clin Pathol 2006;126: 530-533.

28 Rowley JD. Identification of a translocation with quinacrine fluorescence in a patient with acute leukemia. Ann Genet 1973;16:109-112.

29 Estey E, Plunkett W, Gandhi V, et al. Fludarabine and arabinosylcytosine therapy of refractory and relapsed acute myelogenous leukemia. Leuk Lymphoma 1993;9: 343-350.
30 Fenaux P, Lai JL, Preudhomme C, et al. Is translocation $(8 ; 21)$ a 'favorable' cytogenetic rearrangement in acute myeloid leukemia? Nouv Rev Fr Hematol 1990;32: 179-182.

31 Ma SK, Au WY, Kwong YL, et al. Hematological features and treatment outcome in acute myeloid leukemia with t(8;21). Hematol Oncol 1997;15:93-103.

32 Shimada A, Taki T, Tabuchi K, et al. KIT mutations, and not FLT3 internal tandem duplication, are strongly associated with a poor prognosis in pediatric acute myeloid leukemia with $t(8 ; 21)$ : a study of the Japanese Childhood AML Cooperative Study Group. Blood 2006;107:1806-1809.

33 Gilliland DG, Griffin JD. The roles of FLT3 in hematopoiesis and leukemia. Blood 2002;100: 1532-1542.

34 Goemans BF, Zwaan CM, Miller M, et al. Mutations in KIT and RAS are frequent events in pediatric corebinding factor acute myeloid leukemia. Leukemia 2005;19:1536-1542.

35 Dicker F, Haferlach C, Kern W, et al. Trisomy 13 is strongly associated with AML1/RUNX1 mutations and increased FLT3 expression in acute myeloid leukemia. Blood 2007;110:1308-1316.

36 De J, Zanjani R, Hibbard M, et al. Immunophenotypic profile predictive of KIT activating mutations in AML1-ETO leukemia. Am J Clin Pathol 2007;128: $550-557$. 